\title{
Mitteilungen der DGSM
}

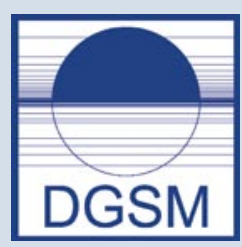

Deutsche Gesellschaft für Schlafforschung und Schlafmedizin Vorsitzender:

Prof. Dr. Geert Mayer, Schwalmstadt-Treysa

DGSM-Geschäftsstelle:

Birgit Tonn-Wilde, c/o Hephata-Klinik Schimmelpfengstr. 2, 34613 Schwalmstadt Tel.: +49-6691/2733, Fax: +49-6691/2823

E-Mail:dgsm.mayer@t-online.de Website: www.dgsm.de
Somnologie $2010 \cdot 14: 244$

DOI 10.1007/s11818-010-0486-5

(c) Springer Medizin Verlag 2010

\section{Anträge auf DGSM-Mitgliedschaft}

Gemäß der am 13.9.2001 in Hamburg aktualisierten DGSM-Satzung werden nachfolgend die Namen der Antragsteller auf DGSM-Mitgliedschaft bekannt gegeben. Erfolgt binnen drei Monaten kein Einspruch durch Mitglieder der DGSM, sind diese Personen automatisch DGSM-Mitglieder: (Stand 10.08.2010)

1. Dr. med. Cornelia Bäuerle, 70199 Stuttgart, Marienhospital Stuttgart

2. Prof. Dr. med. Norbert Dahmen, 56368 Katzenelnbogen, Aarstr. 17

3. Caren Dunkel, 26131 Oldenburg, Hauptstr. 85

4. Dr. med. Alice Engel, 56368 Katzenelnbogen, Aarstr. 17

5. Dr. med. Daniela Ghelfi, CH-8032 Zürich, Steinwiesstr. 75

6. Heike Hein, 35789 Weilmünster, Weilstr. 10

7. Dr. Ina Itzigehl, 50321 Brühl, Rösberger Str. 12

8. Veronika Hupens, 26131 Oldenburg, Hauptstr. 85

9. Cornelia Jung, 70199 Stuttgart, Böheimstr. 37

10. Melanie Kissel, 26131 Oldenburg, Hauptstr. 85

11. Dr. Paul Metz, 57334 Bad Laasphe, Königstr. 4

12. Alexander Radohs, 70199 Stuttgart, Böheimstr. 37

13. Jochen Sauter, 79110 Freiburg, Wirthstr. 17

14. Karin Zemke, 20253 Hamburg, Hoheluftchaussee 18

15. Fariba Zolfaghari, 40670 Meerbusch, Vedasstusstr. 9

\section{Am 13. August 2010 verstarb Prof. Rütger Wever}

Er hatte zusammen mit Prof. Aschoff den bekannten „Bunker“ in Andechs gebaut und führte dort die Langzeitexperimente zur Erforschung biologischer Rhythmen durch. Mit seinen Arbeiten legte er den Grundstock für alle weiteren Studien zu circadianen Rhythmen beim Menschen und fasste dies in seinem Buch "The circadian system of man“, einem Meilenstein der chronobiologischen Literatur, zusammen. Neben dem Einfluß elektromagnetischer Felder befasste sich der Physiker Wever auch mit der Wirkung von hellem Licht auf die biologische Rhythmik. Er war ein wichtiger Förderer der ersten Schlafexperimente in den Freilauf-Studien und trug somit zu der fruchtbaren Zusammenarbeit der Chronobiologie mit der Schlafforschung bei.

Mit Rütger Wever haben wir einen innovativen und hilfsbereiten Wissenschaftler verloren. Als ehemalige Doktoranden des Max-Plank- Instituts für Verhaltensphysiologie haben wir ihm viel $\mathrm{zu}$ verdanken und werden uns gerne an ihn erinnern.

Henner Giedke, Reimer Lund und Jürgen Zulley 